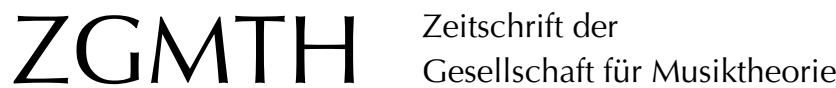

Scheideler, Ullrich (2011): Andreas Jacob, Grundbegriffe der Musiktheorie Arnold Schönbergs, Hildesheim u.a.: Olms 2005 (Folkwang-Studien 1, hg. von Stefan Orgass und Horst Weber). ZGMTH 8/2, 373-380. https://doi.org/10.31751/649

(C) 2011 Ullrich Scheideler

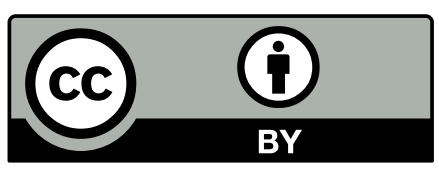

Dieser Text erscheint im Open Access und ist lizenziert unter einer Creative Commons Namensnennung 4.0 International Lizenz.

This is an open access article licensed under a Creative Commons Attribution 4.0 International License.

veröffentlicht / first published: 17/11/2011

zuletzt geändert / last updated: 02/07/2013 


\section{Andreas Jacob, Grundbegriffe der Musiktheorie Arnold Schönbergs, Hildesheim u. a.: Olms 2005 (Folkwang Studien 1, hg. von Stefan Orgass und Horst Weber)}

Das musikalische Denken Arnold Schönbergs fand seinen Ausdruck zeitlebens nicht allein in seinen Kompositionen, sondern stets auch in Formen der schriftlichen Reflexion über Musik. Heterogene Anlässe und Ziele schlugen sich dabei in heterogenen Textsorten nieder: Neben spontan niedergeschriebenen Aufzeichnungen wie kleineren Notizen und Polemiken, deren Zweck insbesondere darin bestand, Aussagen anderer über Musik und Musiker zu kommentieren bzw. zurückzuweisen, und den umfänglichen Manuskripten, an denen Schönberg bisweilen über mehrere Jahre hinweg arbeitete, ehe sie entweder vollendet waren und im Druck erschienen (wie die Harmonielehre) oder aber unfertig liegen blieben (wie die Schrift Komponieren mit selbstständigen Stimmen von 1911 oder das sogenannte Gedanke-Manuskript aus den Jahren 1934-36), entstanden insbesondere anlässlich von Gedenktagen Vorträge über die Bedeutung einzelner Komponisten, sei es für das eigene Schaffen, sei es für den Verlauf der Musikgeschichte (Gustav Mahler, Johannes Brahms, Johann Sebastian Bach), die teils noch zu Lebzeiten des Komponisten publiziert wurden (vgl. die Sammlung Style and Idea von 1950). Nahezu immer dienten Schönbergs Aufzeichnungen der Selbstvergewisserung in Zeiten, in denen das eigene Komponieren in Schwierigkeiten geraten war (so etwa nach 1910, als die Arbeit an der Glücklichen Hand stockte), mit ihnen sollte ferner der eingeschlagene kompositorische Weg erklärt und legitimiert werden. All diese Texte zeigen eine fortwährende Reflexion über Musik als Tonkunst, die für Schönberg im Wesentlichen auf (formalen) Momenten wie Logik, Zusammenhang, Fasslichkeit und Darstellung des Gedankens beruhte. Wie diese Kategorien inhaltlich zu bestimmen seien, war Gegenstand eines geradezu obsessiven Nachdenkens, das sich vermutlich nicht zuletzt durch die Tatsache erklären lässt, dass Schönberg Autodidakt war, sich also mühsam hatte aneignen müssen, was anderen schon früh selbstverständlich geworden war. Eben darum fühlte sich Schönberg in die Pflicht genommen, nicht das zu lehren, was er wusste, sondern das, was seine Schüler nicht wussten (so seine Bemerkung in der Harmonielehre).

In seinem Buch Grundbegriffe der Musiktheorie Arnold Schönbergs, das 2002 als Habilitationsschrift an der Friedrich-AlexanderUniversität Erlangen-Nürnberg eingereicht wurde, hat Andreas Jacob nun den Versuch unternommen, dieses umfangreiche und vielschichtige Material im Hinblick auf die verwendeten musiktheoretischen Grundbegriffe zu gliedern und zu charakterisieren. Da viele Aufzeichnungen Schönbergs bis heute unpubliziert sind ${ }^{1}$, umfasst das Werk neben der "Darstellung" einen weiteren mit "Quellen" überschrieben Teilband, der die teilweise zum ersten Male berücksichtigten Textgrundlagen von Jacobs Darstellung für den Leser zugänglich macht.

Die Arbeit ist in neun Kapitel unterteilt, die jeweils einzelne Momente (respektive Grund-

1 Die Kritische Gesamtausgabe der Schriften Arnold Schönbergs, die derzeit in Wien vorbereitet wird und insgesamt 24 Bände (sowie zusätzliche Kommentar- und Registerbände) umfassen soll, wird den gesamten Bestand öffentlich zugänglich machen. 
begriffe) von Schönbergs musiktheoretischem Denken behandeln. Auf ein knappes Vorwort und ein einleitendes Kapitel (»Schönbergs Musiktheorie als Gegenstand der Forschung «) folgen zunächst drei Kapitel, die sich mit Form und Formenlehre (Kap. II), der Kategorie des musikalischen Gedankens (Kap. III) sowie der Idee musikalischer Logik und dem Postulat der Fasslichkeit (Kap. IV) beschäftigen. Es schließen sich drei Kapitel an, die »Formbildende Prinzipien und Techniken « überschrieben sind. Sie haben Motiv und entwickelnde Variation (Kap. V), Rhythmus und Metrik (Kap. VI) sowie Tonalität und Harmonik (Kap. VII) zum Gegenstand. Die letzten beiden Kapitel sind "Gedankendarstellung in der Kontrapunktik« und »Verdeutlichung des Gedankens als Ziel der Darstellung (Zur Instrumentationslehre) « überschrieben. Die Aufteilung des Quellenbandes verfährt analog.

Jacob nennt als Ziel seiner Studie, „die Schlüsselbegriffe Schönbergs in ihrer Entwicklung und Verortung in Schönbergs System zu beschreiben, wobei die Zusammenhänge der Einbindung in die Lehrtradition bzw. in die zeitgenössische Diskussion« zu berücksichtigen seien (23). Die Formulierung »Einbindung in die Lehrtradition «, die ähnlich bereits zuvor gebraucht wird (17), weist auf eine entscheidende Voraussetzung der Arbeit hin: Jacob geht es nicht (oder allenfalls am Rande) darum, Schönbergs musiktheoretisches Denken mit dessen Komponieren zu verknüpfen. Vielmehr möchte er Schönbergs »originäre[n] Beitrag innerhalb des besagten [musiktheoretischen] Diskurses« (24) genauer bestimmen, d.h. ihn im Kontext bestimmter Lehrtraditionen interpretieren.

Man kann diese Entscheidung problematisch finden, spielen doch in etlichen Schriften Schönbergs eigene Kompositionen eine wesentliche Rolle (vor allem in Composition with Twelve Tones sowie in Heart and Brain in Music). Auch waren Schönbergs kompositorische Erfahrungen unverzichtbar bei der Präzisierung der eigenen Musikanschauung, so dass viele Prinzipien, auf denen Schönbergs Werke beruhen, in seinen Schriften expliziert werden. Und schließlich sind Schönbergs
Lehrbücher oft ausdrücklich als Grundlage eines Kompositionsunterrichts gedacht, in dem die musikalische Analyse, wie wir aus vielen Zeugnissen wissen, zentralen Raum einnahm. Dennoch erscheint Jacobs Entscheidung, auch im Lichte der gegenwärtigen Rezeption, grundsätzlich legitim, insofern Schönbergs Vorstellungen etwa von entwickelnder Variation, von harmonischen Regionen oder von Periode und Satz heute vielfach als (musiktheoretische) Werkzeuge zum besseren Verständnis der Musik von Bachs bis Mahler herangezogen werden. Für Schönberg besaßen bestimmte Prinzipien gleichsam überzeitliche Gültigkeit. Darin, diese Prinzipien herauszuarbeiten, liegt der Sinn der Analyse von sMeisterwerken im Kompositionsunterricht: Sie sollen dem angehenden Komponisten zur zweiten Natur werden, so dass er schließlich ganz seinem Formgefühl vertrauen kann. Die Musiktheorie, und dies ist Gegenstand des zweiten Abschnitts des Einleitungskapitels, liefert die »Kriterien des musikalischen Werts « (35) gewissermaßen nach.

Aus den genannten Voraussetzungen und Vorentscheidungen leitet sich konsequent die Gliederung innerhalb der einzelnen Kapitel ab: Am Beginn steht stets eine knappe Darstellung der Lehrtradition und anschließend die Genese und Entwicklung von Schönbergs Denken über den Gegenstand. Dabei zeichnet sich die Arbeit durch eine starke Quellenorientierung aus. Im Mittelpunkt stehen daher eine zusammenfassende Wiedergabe und Interpretation der Texte sowie ein Vergleich mit anderen Dokumenten, sei es aus dem Umkreis der zeitgenössischen Musiktheorie oder aus anderen Stadien von Schönbergs musiktheoretischem Denken. Bisweilen sind Exkurse eingeflochten.

Das Kapitel über Schönbergs Formdenken beginnt mit der Vorstellung älterer Formenlehren, bei denen Jacob zwei unterschiedliche Richtungen ausmacht: Zum einen sei der Diskurs über Form von der Frage nach dem Zusammenhang bestimmt worden (etwa bei Adolf Bernhard Marx), was unter anderem zur Idee einer organischen Form führte. Zum anderen sei Form in ein Verhältnis zum In- 
halt der Musik gebracht worden, woraus die Vorstellung eines musikalischen Gehaltes erwuchs. Schönbergs »eigentliche Leistung im Hinblick auf die Konstituierung eines tragfähigen musikalischen Formbegriffes" besteht nach Jacob darin, »dass er mit seiner Fassung der Kategorie des musikalischen Gedankens ein Instrument geliefert hat, dass die besagte Entgegensetzung aufzuheben vermochte, indem es ein [...] einheitliches Grundprinzip sowohl von der Formgebung als auch von gehaltlicher Ebene anbietet« (58). Deutlich wird in diesem Kapitel, dass Schönbergs Anschauungen sich nach und nach verschoben haben. Während in den 1910er-Jahren eine eher deskriptive oder vielleicht sogar kasuistische Intention im Vordergrund stand, d. h. lediglich die Merkmale von Formteilen beschrieben wurden, kam es seit den mittleren 20er-Jahren zu einer verstärkten Akzentuierung übergeordneter Prinzipien, die sich zu einer »einheitlichen Theorie« (74) zusammenschließen sollten. Dabei spielten Vorstellungen wie die eines gegliederten Organismus, der Fasslichkeit und der musikalischen Logik eine zentrale Rolle.

Nachdem zunächst ausschließlich auf die technische Seite abgehoben wurde, wird im folgenden Kapitel, der Versuch unternommen, auch die Frage, inwiefern der musikalische Gedanke Träger eines Inhalts bzw. musikalischer Gehalte sein könne, genauer in den Blick zu nehmen. Jacob zeigt zunächst, dass der Begriff des "Gedankens" im musiktheoretischen Schrifttum seit dem 18. Jahrhundert zwei unterschiedliche Entwicklungslinien besitzt. Das »technische« Verständnis verband mit dem Begriff `Thema und 'Motivikı, bisweilen auch den smusikalischen Einfalk. Dem stand ein "ästhetisches" Verständnis gegenüber, das die »Idee« eines Werks, oder die "Essenz des Kunstwerks" meinte; so ist etwa nach Johann Georg Sulzer ein Gedanke dasjenige, „was von einem Werk übrig bleibt«. Schönbergs Verwendung des Begriffs »musikalischer Gedanke« nahm bei zwei Theoretikern des 19. Jahrhunderts seinen Ausgangspunkt, nämlich bei Marx und besonders bei Johann Christian Lobe, wie teils übereinstim- mende oder ähnliche Formulierungen sehr deutlich anzeigen. Im Mittelpunkt standen zunächst das Thema und die Motivik, was allerdings 1911 in der Harmonielehre bereits eine Erweiterung (oder Präzisierung) erfuhr, da mit Thema hier nicht bloß die Melodik gemeint war, sondern auch der musikalische Satz oder die Harmonik. Jacob belegt, wie der Begriff seit den 1920er-Jahren zunehmend ins Zentrum von Schönbergs Musikanschauung rückte. Der »musikalische Gedanke» wurde nun mit der "Grundidee des Werks" gleichgesetzt, wovon die Darstellung des Gedankens abgesetzt wurde. Dabei erfuhr der Begriff nach und nach eine immer stärkere Transzendierung (und wurde als nicht lehrbar erachtet), so dass nur die Mittel der Darstellung als Gegenstand der Musiktheorie, mithin die "Herstellung von Beziehungen" (166) mittels musikalischer Logik übrig blieben.

Damit aber muss Jacobs eingangs geäußerte These, Schönbergs Formdenken führe die Momente des Organischen und des Gehalts zusammen, zumindest relativiert werden. Denn sowohl der musikalische Gehalt selbst als auch das Verhältnis des Gedankens zu seiner Darstellung blieben zu unbestimmt (siehe auch die zitierten Äußerungen von Rudolf Stephan und Horst Weber auf S. 160f.), um für eine Formenlehre praktische Konsequenzen zeitigen zu können. Unübersehbar ist Schönbergs Scheu (vielleicht sogar Unfähigkeit), musikalische Gehalte zu benennen. Die gehaltliche Ebene mag von ihm stets mitgedacht worden sein, als konkret fassbare Formkategorie war sie jedoch untauglich.

Es verwundert daher nicht, dass Jacob sich im Folgenden (im Einklang mit Schönberg) mehr oder weniger ausschließlich technischen Kategorien zuwendet. Das Kapitel über musikalische Logik und Fasslichkeit beginnt mit einem Blick auf die musiktheoretischen Traditionen des erstgenannten Begriffs, wobei erneut zwei Stränge ausgemacht werden, die Schönberg dann zusammenfasste: zum einen der Bereich der harmonischen und metrischen Strukturen (Johann Nikolaus Forkel, Heinrich Christoph Koch, später Hugo Riemann), zum anderen der Bereich der Motivik und The- 
matik. Zunächst spielte der Begriff der Logik bei Schönberg freilich keine Rolle; vielmehr idealisierte er in den Jahren um 1910 das Gegenteil: Unter dem Einfluss einer radikal vertretenen Einfallsästhetik wurde der spontane Ausdruck und (somit) die Unlogik zur zentralen Kategorie des Komponierens. Eine Wende folgte in den 1920er-Jahren, die in der Position von 1934 kulminierte: Jacob legt dar, dass Logik nun als »dritte grundlegende, wenn nicht gar dominierende Bedingung der musikalischen Produktion neben Zusammenhang und Fasslichkeit« (187) trat. Nach Schönberg wurde Logik, die sich in strukturellen Zusammenhängen meist auf der Ebene der motivischthematischen Einheiten zeigt, nicht bewusst hergestellt (siehe etwa seine Ausführungen zur Kammersymphonie op. 9 in Composition with Twelve Tones oder zu Opus 4 in Konstruktives in der Verklärten Nacht). Die Vorstellung, dass das Kunstwerk erschaut, nicht aber konstruiert werden müsse, zählt zu den Grundbedingungen von Schönbergs Komponieren, an denen er über alle Veränderungen hinweg immer festgehalten hat. Logik stellte sich mithin quasi von selbst ein, was aber nur möglich war, wenn das Komponieren durch ein Formgefühl geleitet wurde, das sich wiederum nur erwerben ließ, wenn man die in den Meisterwerken waltende Logik in der Analyse in ihren konkreten Erscheinungsformen studierte. Dasjenige, was Logik ausmacht, änderte sich freilich, und wurde seit den 1930er-Jahren zunehmend mit »Einheit« gleichgesetzt. Einheit aber entsteht durch Zusammenhang, und ein wahrnehmbarer Zusammenhang ermöglicht gleichzeitig Fasslichkeit - eine Kategorie die ebenfalls spätestens seit den 1930er-Jahren zunehmend an Bedeutung gewann.

In drei Abschnitten befasst sich Jacob mit dem Verhältnis von 'Logikı und Dodekaphonie, mit der Funktionsweise der Logik sowie mit `Logikı als Bedingung einer Kompositionslehre, um zwei weitere Abschnitte anzuschließen, die den Begriff der Fasslichkeit in den Blick nehmen (»Funktionsweise und formale Implikationen«, „Fasslichkeit als dynamisches System«). Gezeigt wird unter anderem, dass Schönberg zunehmend dazu neigte, Fasslich- keit unter Zuhilfenahme des Ausgleichsprinzips zu begreifen (bzw. in Kompositionen zu erzielen), womit er sich in gewisser Weise in einen Widerspruch zu der Idee setzte, dass »eine dem Gedanken adäquate (meist als ıdirektı oder sgedrängt beschriebene) Darstellungsform in mehreren Dimensionen gleichzeitig anzustreben sei« (232). Die Darstellung des Gedankens nahm also zunehmend den Zuhörer in den Blick, sollte sich an seinen Auffassungsmöglichkeiten orientieren (eine Vorstellung, die von Schönberg und Erwin Stein als Argument herangezogen wurde, um zu erklären, warum dodekaphone Werke sich alter Formen bediente). Jacob betont daher zu Recht, dass »Fasslichkeit [...] als Regulativ künstlerischer Produktion gesehen « werde (241).

In den drei folgenden Kapiteln, die »Formale Prinzipien und Techniken« überschrieben sind, wird nun die Bedeutung derjenigen Mittel für Schönbergs Musiktheorie untersucht, die der Schaffung von Zusammenhang dienen. Das erste dieser Kapitel beschäftigt sich mit der Rolle, die das Motiv und die entwickelnde Variation im musiktheoretischen Denken Schönbergs spielen. In Schönbergs Schriften änderte sich allmählich die Auffassung davon, was als Motiv bezeichnet werden kann, (was - wie Jacob dargelegt - auch mit unterschiedlichen Definitionen von Motiv zusammenhängt, die seit der Mitte des 19. Jahrhunderts im Umlauf waren). Zwar benennt Schönberg von Beginn an das Moment der Wiederholung (und der Variation) als grundlegend, doch wechseln diejenigen Parameter, auf die sich diese Wiederholung bezieht. 1917 war es noch die Rhythmik (und somit ein stark an der musikalischen Oberfläche wirkendes Moment), später treten Rhythmus und Diastematik gleichberechtigt nebeneinander. Vielleicht hätte Jacob hier noch weiter gehen können, denn ein Aufsatz wie Brahms the Progressive (sowohl in der Fassung von 1933 als auch besonders in der publizierten Fassung von 1947) oder auch die Ausführungen in Fundamentals of Musical Composition heben beim Motiv vor allem auf das Moment der Diastematik ab, die als Zusammenhang 
stiftendes Moment gleichsam im Hintergrund wirken soll. Neben technischen Bedingungen eines Motivs (z. B. Länge, Gestalt) werden im Folgenden auch die Metaphern zu dessen Beschreibung untersucht, zum einen - an Früheres anknüpfend - die Unterscheidung zwischen Gedanke als etwas Geistigem und Motiv als etwas Materiellem (zur Darstellung dieses Gedankens), zum anderen die Vorstellung, das Motiv sei der Keim eines Werkes. Jacob kann anhand von Dokumenten anschaulich zeigen, dass Schönberg um 1910 durchaus der Vorstellung von Motiv als Keim anhing (zumindest bei älteren Sinfonien, vgl. S. 265). In späteren Äußerungen und im Hinblick auf sein eigenes Schaffen wurde diese Auffassung indes zurückgewiesen, und ihr allenfalls der "Wert eines Bildes, eines Vergleichs" (267) zugebilligt. Auch diese Idee Schönbergs hing mit der Vorstellung vom musikalischen Gedanken zusammen, der als Ganzes erschaut werde, während das Motiv nur das Darstellungsmittel abgebe. Die Vorstellung des Organischen wurde (um 1936) verabschiedet, weil sie zu sehr mit der Vorstellung verbunden sei, das Wachsen geschehe gleichsam von selbst. Stattdessen betonte Schönberg nun die eigenständige Leistung und Freiheit des kompositorischen Subjekts.

Ein längerer Abschnitt befasst sich mit dem Verhältnis von Motiv, Wiederholung und entwickelnder Variation. Die Auffassung, dass Wiederholung und Abwechslung in einer Art von Balance stehen müssten, änderte sich nicht. Dagegen wechselte die Begründung, die später eine stärkere geschichtsphilosophische Akzentuierung erfuhr, in der eine (unveränderte) Wiederholung als Zeichen der Kunstlosigkeit galt. Zugleich wird unterschieden, ob Varianten komponiert werden, um (bloße) Abwechslung zu erreichen (quasi ornamental), oder aber eine zielgerichtete Entwicklung nehmen. Damit rückte die Idee von entwickelnder Variation ins Zentrum von Schönbergs Anschauung, ganz im Einklang mit der Vorstellung von Logik und Zusammenhang. Die Verfahren entwickelnder Variation grenzt Jacob von denen motivischer Arbeit und Sequenz ab, die aufgrund ihrer mangeln- den Zielgerichtetheit bzw. der Mechanik von Schönberg als minderwertig (und historisch überholt) beurteilt werden.

Das Kapitel über Rhythmus und Metrik geht zunächst Schönbergs Versuchen nach, den Begriff Rhythmus zu definieren und in ein Verhältnis zu anderen Begriffen, insbesondere zu Motiv und Metrik zu setzen. Dazu werden größere syntaktische oder musikalische Einheiten (Gestalt, Phrase) erörtert, die nur mittelbar oder teilweise mit dem Hauptbegriff zusammenhängen. Am Ende folgen zwei Abschnitte über Metrik und musikalische Prosa. In diesem Kapitel, dessen Hauptüberschrift ja »Formbildende Prinzipien und Techniken" lautet, hätte man erwartet, dass eine Untersuchung des Rhythmus- und Metrikbegriffs bei Schönberg wieder wesentlich unter dem Blickwinkel von musikalischer Logik, Fasslichkeit, Zusammenhang und entwickelnder Variation erfolgen würde. Dies ist gleichwohl nur verhältnismäßig spärlich der Fall, da Schönberg sich vor allem mit der Klassifikation von Rhythmen sowie möglichen Verhältnissen von rhythmischen Einheiten zur Taktmetrik beschäftigt. Zu einer detaillierten Ausarbeitung der Rolle, die der Rhythmus für die entwickelnde Variation spielte, kam es nur am Rande. Schönberg konzentrierte sich stattdessen auf unregelmäßige Akzentfolgen, was Jacob veranlasst, der Idee von Phrase und später von musikalischer Prosa, also einer metrischen Unregelmäßigkeit auf einer höheren Ebene nachzugehen.

Ausführlich vorgestellt werden Schönbergs Auffassungen, wie sie 1934 im Gedanke-Manuskript zu finden sind. Schönberg ging hier soweit, die metrische Qualität des Taktes zu leugnen (oder mindestens zu vernachlässigen) und - in Jacobs Worten - »in der Taktnotation überhaupt nur ein Mittel visuell sinnfälliger Zusammenfassung, das keine apriorischen Konsequenzen für die Akzentgebung nach sich ziehen muss", zu erkennen (367). Damit ist indes die entscheidende Frage noch nicht gestellt, nämlich ob bei »Betonungsverschiebungen innerhalb der Takte « und der Darstellung "jedwede[r] rhythmische[r] Feinheit» (366) bzw. bei der Tatsache, »dass Betonungs- 
verhältnisse nicht stereotyp durch den Takt bestimmt $[\ldots]$, sondern in ein individuell austariertes Beziehungsgeflecht» (368) gebracht werden, die Taktmetrik weiterhin im Hintergrund spürbar bleibt (so dass es - ganz traditionell - zu einer Spannung zwischen melodischem und metrischem Akzent kommt) oder aber die Taktmetrik sich tatsächlich auflöst und somit als Hintergrundfolie nicht länger wirksam ist. Hier wäre sicher eine Diskussion von Schönbergs eigenen Werken hilfreich gewesen, d.h. der Frage, ob etwa dem Bläserquintett op. 26, den Quartetten op. 30 und 37 oder den Variationen für Orchester op. 31 es Schönberg wirklich gelungen ist, eine freie Taktmetrik zu komponieren oder aber doch die (unregelmäßigen) melodischen Akzente ihre ästhetische Qualität (vor allem) durch ihrer Abweichung von der (regelmäßigen) Taktmetrik erhalten. Schönbergs Bestreben, Bach, Mozart oder Brahms als Vorbild für eine freie Taktmetrik in Anspruch zu nehmen (vgl. etwa S. 362, S. 368 oder S. 370), hätte einer ausführlichen Prüfung, respektive Diskussion bedurft.

Das letzte Kapitel im Teil »Formbildende Prinzipien und Techniken « wendet sich der Tonalität und der Harmonik zu. Jacob beginnt mit der Bemerkung, dass die Beschäftigung mit der Tonalität angesichts des eigenen (kompositorischen) Übergangs zur Atonalität doch verwunderlich und die Harmonielehre unter anderem als »Rechtfertigungsinstanz gegen etwaige Anwürfe des Dilettantismus« (374) zu bewerten sei. Beides aber erscheint eine zumindest einseitige Interpretation, denn im Hinblick auf die Auffassung, dass das Verstehen der Meisterwerke eine wesentliche Voraussetzung für das Komponieren darstelle, mussten eben auch die Kategorien erarbeitet werden, die ein adäquates Verstehen ermöglichten - und dazu gehörten zweifellos die Regeln der Akkordverbindungen. Jacob kann im Folgenden anschaulich zeigen, dass Schönbergs Argumentation, Tonalität trage lediglich zur Formbildung bei, stelle aber keine unumstößliche Instanz der Musik dar, sondern könne vielmehr durch andere Momente ersetzt werden, sich als erstaunlich sta- bil erweist. Flankiert wurde diese These von einem geschichtsphilosophischen Modell, nach dem das Tonsystem zur Vereinfachung tendiere (von den Kirchentonarten zum DurMoll-tonalen System zur chromatischen Tonart; S. $396 \mathrm{ff}$.), wobei die Musik Bachs und Wagners als Kronzeuge herangezogen wird. Damit war für Schönberg auch beim Tonsystem ein Modell gefunden, das die Einheit des Werks verbürgen könne, und das er in der 12-Ton-Methode als »einheitlich aufgespanntem Raum« (443) verwirklicht sah: die von ihm so genannte sMonotonalitäts. Jacob zeigt abschließend, dass mit dem Begriff Tonalität zwei Momente in Schönbergs Denken verbunden waren: einerseits, dass Tonalität als natürliches Resultat aus dem Wesen des Tons folge, und andererseits, dass Tonalität durch Kunstmittel erzeugt werde und ihre Leistung durch andere Mittel ersetzt werden könne.

Das vorletzte Kapitel ist mit »Gedankendarstellung in der Kontrapunktik« überschrieben. Schönberg hat sich im Laufe seines Lebens in ziemlicher Regelmäßigkeit mit kontrapunktischen Verfahrensweisen und adäquaten Lehrmethoden beschäftigt. Ausgangspunkt der Darstellung ist die Prämisse, dass zwei Momente unterschieden werden müssen: einerseits konkrete kontrapunktische Kompositionen (insbesondere Johann Sebastian Bachs), andererseits der »reine« Kontrapunkt als Gegenstand der Propädeutik (oft im Rückgriff auf Johann Joseph Fux). Diese doppelte Bestimmung hat, wie in einem Exkurs ausgeführt wird, eine lange Lehrtradition. Eine wohl doch wesentlichere Unterscheidung wird von Jacob allerdings nicht getroffen: zum einen die Möglichkeit, den kontrapunktischen Satz von der Zweistimmigkeit her zu entwickeln - so die Fux-Tradition - zum andern von der Vierstimmigkeit auszugehen, somit gleich die Harmonik mitzudenken - so die Kirnberger-Tradition, die bei Marx ihre Fortsetzung findet. Das Verhältnis von Stimmführung und Harmonik ist aber, wie Jacob dann bereits an Schönbergs Fragment Komponieren mit selbständigen Stimmen (1911) zeigt, eine immer wieder diskutierte Frage. Zunächst betonte Schönberg sehr stark das Moment der Selbständigkeit der 
Stimmen, wenn er die Harmonik als Regulativ bezeichnete, das nur der »Kontrolle, Geschmackskontrolle« (465) diene. Auch 1917 wurde noch vor allem die Unabhängigkeit der Stimmen hervorgehoben. Im Laufe der Zeit trat zum einen die Frage des Motivs ins Zentrum von Schönbergs Überlegungen (alle Gestalten sollen, unabhängig davon, ob sie Thema oder Kontrapunkt sind, sich auf dasselbe Motiv rückbeziehen lassen), zum anderen damit zusammenhängend - und dies scheint der eigentlich originelle Gedanke Schönbergs zu sein - die mit Kontrapunkt verbundenen Formen, die sich von Formen mit anderen Satztechniken unterscheide. Entwickelnde Variation sei das »Formprinzip der homophonmelodischen Kompositionsmethode« (477), der kontrapunktische Satz zeichne sich hingegen durch das Verfahren der Abwicklung aus. Jacobs (teilweise Schönbergs Begriffe einbeziehende) Formulierung, das "Grundmotiv der kontrapunktischen Arbeit unterliegt keiner logisch verfahrenden Entwicklung, sondern enthält idealerweise salle zukünftigen Gestalten bereits in sich, so dass sie sohne weitere Hinzufügung, ohne Entwicklung [...] aus ihm herausgeschält werden können« (477) klingt dann aber sehr nach einer Anschauung, die zuvor verworfen worden war, nämlich der Keim-Metapher. Insofern wäre beim Motivbegriff stärker (nach Satztechnik und vielleicht auch chronologisch) zu differenzieren. In den folgenden Jahrzehnten blieben die beiden Fragen nach der Einheitlichkeit des musikalischen Materials und nach dem Verhältnis von Kontrapunktik und Harmonik gleichermaßen aktuell, so dass mal mehr das eine, mal mehr das andere akzentuiert wurde. Für die Auffassung, dass durch die Emanzipation der Dissonanz die Stimmen kein determiniertes Auflösungsbestreben mehr besäßen, und dass Kontrapunkt die Kunst sei, Tongestalten zu erfinden, die sich selbst begleiten können, wurde dabei bis in die 1930er-Jahre stets Bach als Vorbild bemüht. Jacob legt dar, dass nach 1933 jedoch eine recht deutliche Neuorientierung erfolgte, die nun Fux und die Propädeutik ins Zentrum rückt. Damit war gleichzeitig eine Ablehnung des Begriffs »linearer Kontra- punkt« verbunden. Beides war Ausdruck einer neuen Akzentsetzung, die im Angesicht auch aktueller Strömungen der zeitgenössischen Musik erfolgte und sich gegen die Vernachlässigung des Zusammenklangs wandte.

Das letzte, eher knapp gehaltene Kapitel beschäftigt sich mit der Instrumentationslehre. Auch hier entdeckt Jacob bei Schönberg das Zusammenführen zweier Prinzipien: zum einen der Idee der Emanzipation der Klangfarbe als Parameter eigenen Rechts, zum anderen der Auffassung, dass die Funktion der Instrumentation darin bestehen müsse, die Struktur des Tonsatzes deutlich zu machen. Bei Schönberg folgte aus diesen beiden Prämissen zum einen der Grundsatz der Transparenz des Klangs, zum anderen (und in erster Linie) die Vorstellung, dass sich Satzart und gewählte Instrumentation aufeinander unmittelbar beziehen müssen. Eine Instrumentationslehre muss sich also nicht allein mit dem Klang als Farbe beschäftigen; vielmehr müsse die Ausgangsfrage - in einer Formulierung Schönbergs - lauten: "Wie ist ein Satz zu erfinden, der von einer bestimmten Art und Zahl von Instrumenten gespielt werden soll?« (536). Die Anbindung der Instrumentation an die Satzlehre, die (wie Jacob zeigt) auf Lehrtraditionen durchaus zurückgreifen kann, wurde mit dem Ideal der Ökonomie verbunden, die Schönberg allein in der Sinfonik Gustav Mahlers realisiert sah. Somit wurde eine »Koloristik, die ohne strukturelle Funktion eingesetzt wird« (543), abgelehnt.

Andreas Jacobs Studie über die Grundbegriffe der Musiktheorie Arnold Schönbergs ist zweifelsohne eine akribische Arbeit, die auf umfangreichen Quellenstudien fußt und eine Fülle von Literatur über Schönberg einbezieht. Schon der Umfang von über 1000 Seiten (der Darstellungsteil umfasst 550 Seiten, der Quellenteil etwa 400 Seiten, hinzu kommt ein Literaturverzeichnis von 100 Seiten Länge) demonstriert eindrucksvoll die umfassende und viele Details einbeziehende Qualität dieser Arbeit.

Warum aber macht sich bisweilen dennoch ein gewisses Unbehagen breit? Neben einigen formalen Kleinigkeiten (nicht gut gekennzeich- 
nete Auslassungen bei Zitaten, diverse Trennfehler, teilweiser Verzicht auf Korrektur von offensichtlichen Tippfehlern in Typoskripten Schönbergs) scheinen zwei Gründe hierfür verantwortlich. Zum einen vermisst man die große Linie. Es fällt schwer, den Überblick zu behalten, oft ändern sich nur Nuancen (etwa bei der Auffassung vom Motiv), manchmal gehen radikale Brüche im Fluss der Darstellung unter. Dazu trägt auch bei, dass ein Sach- und Personenregister fehlt, welches eine bessere Orientierung bieten und Brücken über die Kapitelgrenzen hinweg schlagen könnte. So sind etwa die unterschiedlichen Aussagen, die Schönberg über Bach und seine Zeitgenossen (Händel, Telemann) macht (insbesondere im Quellenteil gibt es hierzu eine Reihe von Äußerungen, vgl. etwa S. 758 ff. oder S. 820) interessant, weil sie das Besondere von Schönbergs Melodie- und Kontrapunktbegriff und somit Grundlagen seiner Musikanschauung schlaglichtartig klarmachen. Zum anderen (und dieser Punkt ist von größerer Bedeutung) bleibt die Darstellung aus zwei Gründen nicht selten recht unanschaulich. Erstens wird auf Notenbeispiele durchgängig verzichtet, und zwar selbst dort, wo im Original bei Schönberg Notenbeispiele notiert sind (im Quellenteil folgt dann entweder der pauschale Hinweis »folgt Notenbeispiel« oder aber die Mitteilung, um welchen Ausschnitt etwa eines klassischen Werkes es sich handelt). Wer aber die gedruckten Aufsätze oder Bücher wie die Harmonielehre oder Fundamentals of Musical Composition kennt, wer die Entwürfe Zusammenhang, Kontrapunkt, Instrumentation, Formenlehre (1917) oder Der musikalische Gedanke und die Logik und Technik und Kunst seiner Darstellung (1934-36) zur Hand nimmt, weiß, dass erst die Notenbeispiele das theoretisch Erläuterte zur deutlichen Anschauung bringen. Zweitens stellt sich bisweilen unwillkürlich die Frage, welche Konsequenzen denn aus Schönbergs Definitionen erwachsen, sei es im eigenen Komponieren (das Jacob ja aus einsehbaren Gründen weitgehend außen vor lässt), sei es bei der Lehre im Kompositionsunterricht. Auch dies hätte die Anschaulichkeit sicher erhöht. So gerät die Lektüre der Arbeit mühsamer als es vielleicht hätte der Fall sein müssen.

Dass die Arbeit neue Fragen aufwirft, ist freilich kein Mangel, sondern darf umgekehrt als einer ihrer Vorzüge bewertet werden. Jacobs Studie kann sowohl durch den Darstellungsteil als auch die zahlreichen Quellen im zweiten Band als gewichtiges Fundament dienen, auf dem nun Detailstudien aufbauen können. Damit ist sie eine Grundlagenarbeit im besten Sinne.

Ullrich Scheideler 